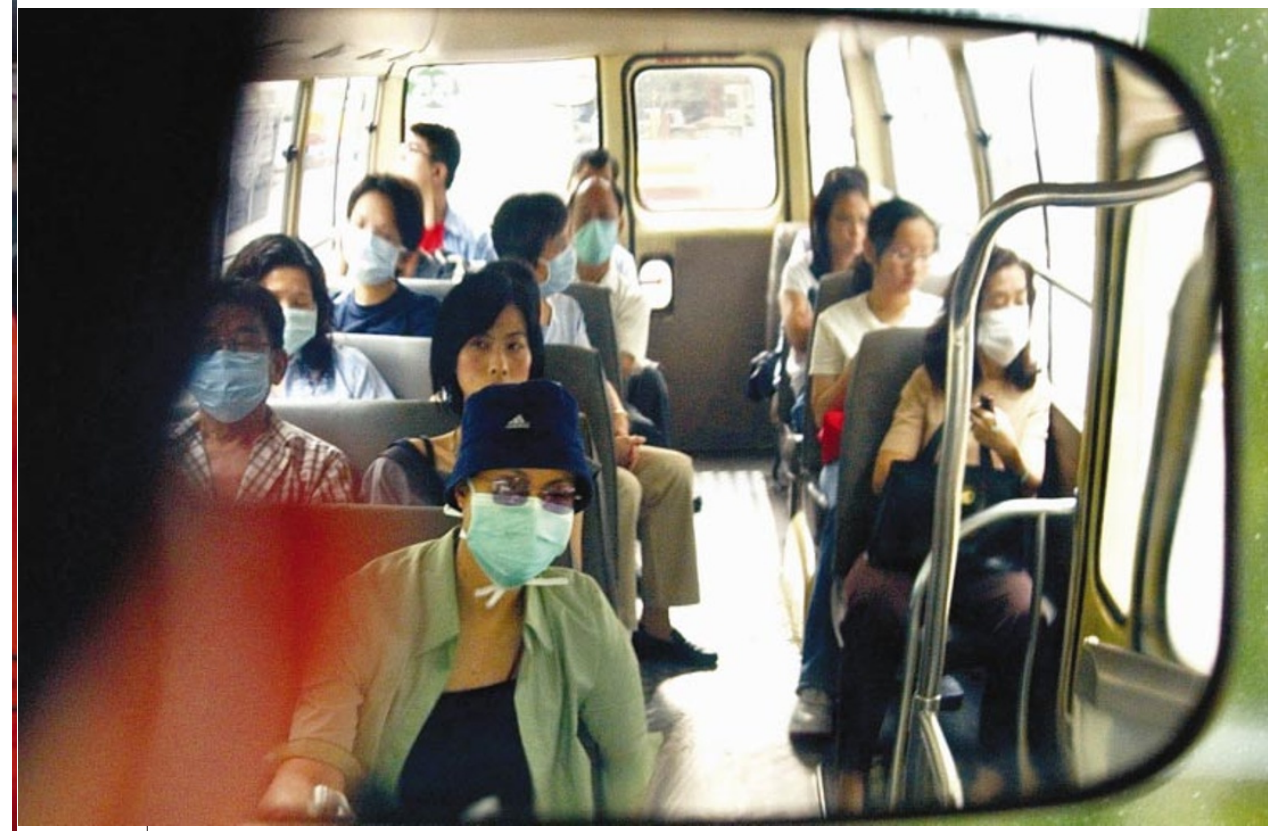

Triumph and tension: a varied year for China saw Yang Liwei (left) feted as a national treasure after becoming the country's first man in space, while on the ground panic spread over the SARS epidemic.

concerned about safety. "We are really worried about the risk of SARS escaping from a laboratory," says parasitologist Zhao-Rong Lun of Zhongzhan University in Guangzhou.

Given these problems, wholesale changes are needed before China can truly claim a place among the world's premier scientific, medical and technological powers. The SARS story is symptomatic of a wider failure to tackle public-health issues. Hepatitis B is rampant and AIDS is a growing problem, particularly in Henan province, where tens of thousands of people are thought to have become infected in the 1990s thanks to poor hygiene in a scheme for collecting blood plasma. 'Western' diseases of affluence are also on the rise - obesity among schoolchildren, for example, has risen tenfold over the past eight years.

Meanwhile, China's burgeoning research purse is not matched by a corresponding development of structures to ensure that funds are distributed
"Wholesale changes are still needed before China can claim a place among the world's premier scientific powers." meeting highlighted the problems of datafalsification and plagiarism - bold stuff in a country that, until recently, would never have admitted that such misdemeanours take place. Some scientists remain frustrated, however, at the lack of concrete progress towards solving these problems. "Things are not changing fast enough," laments C. L. Tso, a retired molecular biologist who has been pushing for reform for the past 15 years.

Others wonder whether China has its priorities right. Says one molecular biologist at the Shanghai Institutes for Biological Sciences: "Can we really afford a space programme when we have so many people sick and lacking medical care?"

Nevertheless, scientists who have made their names abroad are flooding back to China. Their reasons are many and varied: some are enthused by the chance to run their own research group at a young age; others say they want to contribute to China's by merit, or that ongoing projects are properly evaluated. Cronyism is rife, researchers complain. "I constantly have to fight a system that is not used to a merit-based approach," says $\mathrm{Mu}$ ming $\mathrm{Pu}$, who divides his time between the University of California, Berkeley, and his post as director of the Chinese Academy of Science's Institute of Neuroscience in Shanghai.

There is a growing recognition that reform is needed. In September, at a meeting of the China Association of Science and Technology, a semi-governmental body that oversees China's various academic societies, its chair Guangzhao Zhou criticized health officials' unsupported claims about the cause of the SARS outbreak. Presentations at the development; some are undoubtedly enticed by lavish salaries and research funding.

Botanist DeZhu Li was an early returnee. In 1997 he joined the Kunming Institute of Botany in southwestern China, after three years of postdoctoral work in Britain. He was quickly made deputy director of the institute, which will soon open a new, US\$18-million germplasm bank to store seeds and frozen tissue samples. "It's a really good sign," says Li. "The government cares about science and it has the resources to back this up."

\section{David Cyranoski}

\footnotetext{
1. Chen, Y. et al. Cell Res. 13, 251-263 (2003)

2. Yang, H. et al. Proc. Natl Acad. Sci. USA 100, 13190-13195 (2003). 3. Zhong, N. S. et al. Lancet 362, 1353-1358 (2003).
}

\section{HIGHLIGHTS}

\section{History on ice}

A core some 3.2 kilometres long was pulled from the Antarctic ice, allowing geologists to see at least 750,000 years into the past. Although not the longest ice core ever recovered, it is the oldest, and should contain a record of eight ice ages. Palaeoclimatologists are especially keen to inspect a period some 450,000 years ago when Earth's orbit was very similar to what it is today, giving a good idea of what the present climate might have been like without human influence.

\section{Copy rats}

Rats have been a favourite lab animal since the early nineteenth century and, for many studies, provide a better physiological match with humans than mice do. But they missed out on the cloning revolution of the 1990s - the method that gave us cloned mice failed to work. This year researchers came up with a subtly different technique, which involves delaying the activation of the egg, to clone the rodents successfully. Using cloning, it should become easier to produce certain types of genetically engineered rat.

\section{Puzzle solved?}

A Russian mathematician claimed to have proved the Poincaré Conjecture, a problem that has gone unsolved since 1904, and which involves properties of objects that do not change when they are stretched or shrunk - such as their number of surfaces. Grigori Perelman of the Steklov Institute of Mathematics in St Petersburg toured American universities describing his solution. Something of an eccentric, he does not plan to submit his work to a peer-reviewed journal.

\section{Flavour saviour}

Particle physicists confirmed the mechanism by which the ghostly particles known as neutrinos flicker between different forms, or 'flavours', with an elegant experiment. Japan's KamLAND detector on the island of Honshu picked up neutrinos belted out by a ring of nuclear reactors that coincidentally surround it. Previous experiments have relied on the Sun as a neutrino source. Although physicists have a good idea of the number and type of neutrinos emitted by the Sun, there remained a tiny uncertainty, which the KamLAND experiment neatly eliminated.

\section{Quantum leap}

The second of the two basic building blocks of a quantum computer - a 'controlled NOT' logic gate, which flips the state of a target bit of information based on the state of a control bit was demonstrated by several teams this year. One group managed to make the gate in a solidstate system, using electrons in a silicon chip. The same team also made a solid-state version of the first building block - a 'rotation' gate in 1999. But no one has yet linked the two gates together to form a functioning computer. 